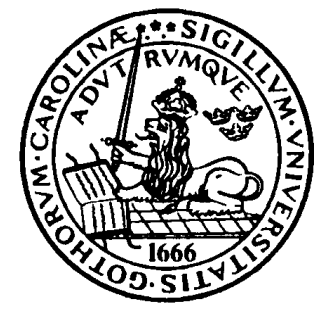

Nuclear Instruments and Methods in Physics Research A 357(1995)129-149

\title{
Track reconstruction in the ATLAS detector using elastic arms
}

\author{
Magnus Lindström \\ Physics department \\ Lund University \\ Sölvegatan 14 \\ S-223 62 Lund \\ Sweden \\ email:jml@quark.lu.se
}

\begin{abstract}
The elastic arms algorithm for track reconstruction is implemented on the proposed ATLAS detector at LHC. New features are two parameterizations of tracks in fields, an improved Hough transform and the use of Hessian matrices for updating. The handling of hit ambiguities is incorporated in the algorithm. The results are well in accordance with the theoretical expectations.
\end{abstract}




\section{Introduction}

To be able to explore new energy regions, CERN plans to build a new proton collider, the LHC. It will collide protons, of which each will have an energy of 7 $\mathrm{TeV}$. This will make it possible to study unexplored parts of the standard model, and possibly discover new areas of physics.

The cross section for point like reactions decreases with the square of the energy, so the probability of obtaining interesting reactions at LHC energies will be small. This forces the LHC to run at an extremely high luminosity to be able to obtain reasonable statistics. Even at a minimum bias collision frequency of $10^{9}$ collisions per second, there will, according to [1], only be about 10 Higgs events per year if the Higgs mass is $1 \mathrm{TeV}$.

At the LHC energies there will typically be about 30 particles per event, but there will be 20-30 events at the same time in the detector.

These circumstances, rare events in a noisy environment, put even higher demands on the detector than in previous experiments.

In the tracking detector, position measurements are made along the particle trajectories. In the ATLAS detector there will be many hits (position measurements), also from background, which will make the track reconstruction very difficult to accomplish.

The track reconstruction is a two-step problem. The first step is the task of deciding which hits were made by a specific particle, the second is the problem of finding the optimal set of track parameters for these hits. Normally these problems are solved separately, but at LHC the track density is going to be so high that this might not be feasible. In this article a novel algorithm, the elastic arms algorithm, derived by M. Ohlsson et al. in [2], is studied for simulated ATLAS data. It combines the two steps by the use of neural decision units, and thereby supplies a global formulation. The neural decision units operate with probabilities in the sense that they assign a hit to the track that it most probably belongs to. These assignments can in extreme situations be made at a very late stage of the fitting process and thereby makes it possible to avoid any forced premature decisions.

This article starts with a presentation of the tracking detectors of ATLAS in section 2. Two new parameterizations of tracks are derived in section 3.1, one exact and one approximate which is faster. To make the parameterizations versatile the detector characteristics are incorporated in a general way. The local Hough transform [3], which is used to initialize the algorithm, is discussed in section 3.2, and the transform is adapted to the actual detector resolution. The algorithm is discussed in section 3.3 and as suggested in [4] the Hessian matrix [5] is used for updating. A way of computing these matrices in a fast manner is derived in appendix B. The fact that particle trajectories intersect in the event vertex makes it necessary to treat this point in a special way. A solution is derived and used with the exact parameterization. The handling of ambiguities 
is incorporated in the algorithm in the way derived by R. Blankenbecler in [6]. The algorithm is tested on simulated data from the ATLAS TRT detector.

\section{The ATLAS detector}

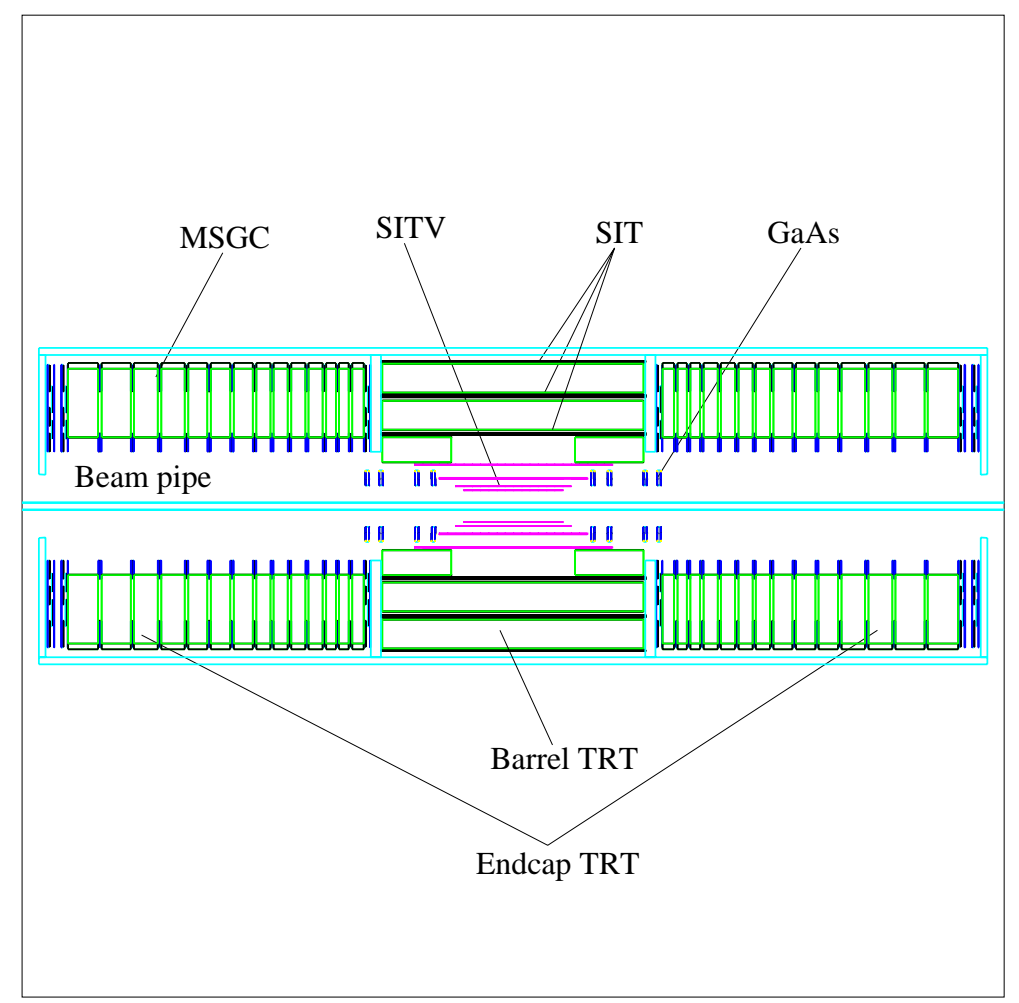

Figure 1: The inner detector of ATLAS.

The ATLAS detector is a general purpose experiment that is proposed to be operational at the start up of the LHC.

The design consists of two major parts. The inner detector, used for track reconstruction purposes, and the outer calorimeter part, used for energy measurements and particle identification.

The part that is of interest in this article is the inner detector (see figure 1). It consists of five different kinds of subdetectors

1. TRT, transition radiation detector/tracker, gives position measurement both in the endcap and barrel region by straw-drift tubes and the transition radiation detector is used for electron identification.

2. MSGC, microstrip gas chamber, gives precision measurement in the endcap region. 
3. SITV, silicon inner tracker/vertex, which purpose is to determine the position of the primary and secondary vertices.

4. SIT, silicon tracker, gives track data with $95 \mathrm{~cm}$ long strips in the barrel region. It only supports the TRT.

5. GaAs, gallium arsenide, improves position measurement in the forward region.

The inner detector is enclosed by a superconducting solenoid which gives a magnetic field of $2 \mathrm{~T}$ parallel to the beam pipe. This magnetic field forces the charged particles to follow a curved trajectory in the plane perpendicular to the field.

The track finding is going to take place in the TRT, where the straw-drift tubes provide the position measurement. The readout information consists of the position of the straw which was penetrated and the distance from the anode to the closest point where the charged particle passed. The straws are $4 \mathrm{~mm}$ in diameter and the tracking is done with a precision of $150 \mu \mathrm{m}$.

- The barrel detectors cover an axial length of $2 \times 95 \mathrm{~cm}$, between 60 and 95 $\mathrm{cm}$ in radial direction. The straws have a uniform radial spacing of $8 \mathrm{~mm}$ between them. The two detectors each have $130 \cdot 10^{3}$ readout channels.

- The endcaps are, as their name implies, positioned at the end of the barrels and have their straws positioned radially (wheels of straws) from 50 to 100 $\mathrm{cm}$. They cover an axial section from 100 to $330 \mathrm{~cm}$.

The part used here is the barrel detector.

\section{Algorithm}

In this section, the track reconstruction algorithm is developed and discussed. It starts in 3.1 with a discussion about charged particles in magnetic fields and parameterization of their movement in the same. The Hough transform, which is used to initialize the elastic arms algorithm, is discussed in 3.2. The algorithm is presented in 3.3 without any proof (this is given in appendix A). The section is concluded with a description of the program used.

Some definitions are needed to be able to understand this section.

- A track is the particle trajectory that we want to reconstruct. In the detector a number of hits are left as a trace and these are used to reconstruct the track.

- An arm is a parameterization of an ideal track. This is possible since the ideal particle trajectory is known. The task is to fit the arms to the tracks.

Index $a$ is used for arms, and $i$ or $j$ for hits. 


\subsection{Parameterization}

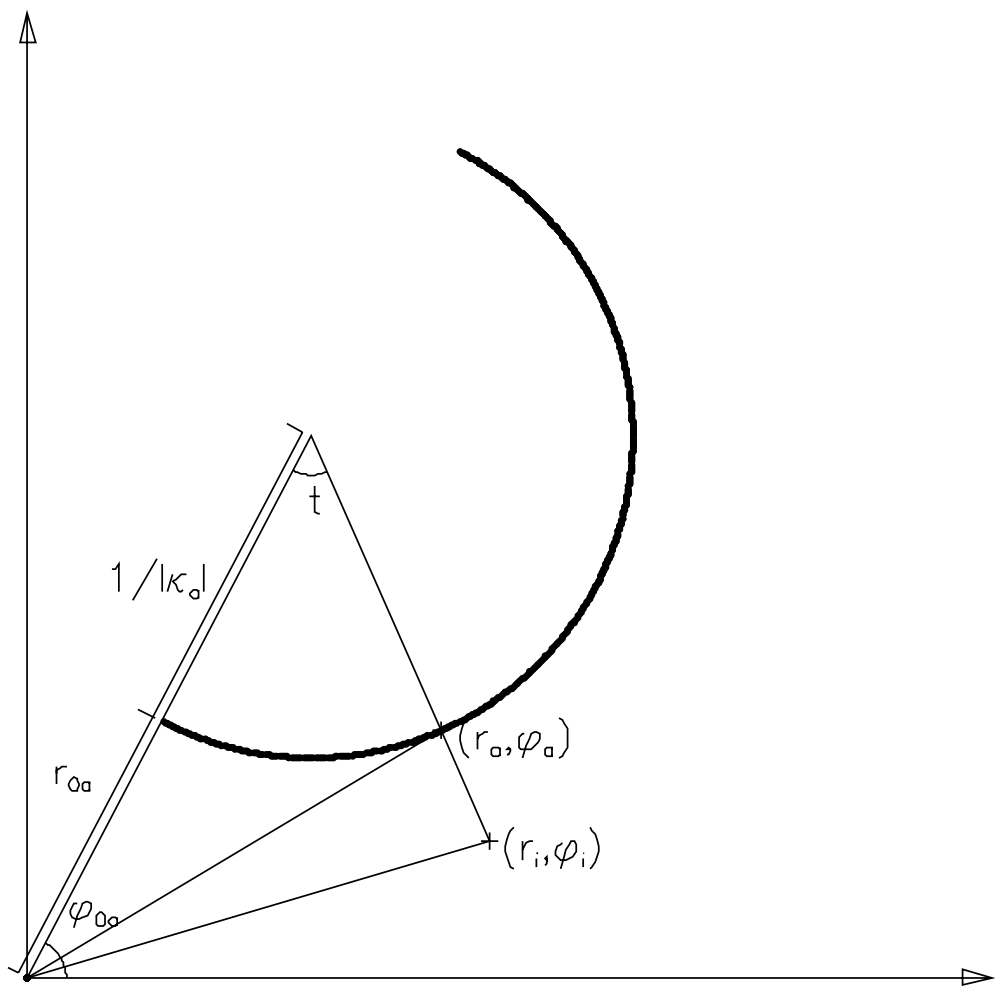

Figure 2: Parameterization of a track in the transversal plane. $\left(r_{a}, \phi_{a}\right)$ is the point on $\operatorname{arm} a$ which is closest to hit $i$.

A charged particle which moves in a magnetic field perform a circular motion in the plane that is perpendicular to the field. The momentum component that is along the magnetic field will be left unchanged. These two components, normally denoted as transverse momentum for the circle and longitudinal for the translation along the beam pipe, gives a helix in space. For a particle with unit charge the relation between the magnetic field $B[T]$, and the inverse radius $\kappa\left[m^{-1}\right]$ is

$$
p_{t}=\frac{0.3 B}{\left|\kappa_{a}\right|} \quad[\mathrm{GeV} / \mathrm{c}]
$$

The detector geometry makes it natural to choose cylindrical coordinates for the parameterization. A helix in three dimensions with the magnetic field along the $\mathrm{z}$-axis is given by

$$
r_{a}=\sqrt{\left(r_{0 a}+\frac{1}{\kappa_{a}}(1-\cos (t))\right)^{2}+\left(\frac{1}{\kappa_{a}} \sin (t)\right)^{2}},
$$




$$
\begin{gathered}
\phi_{a}=-a \cos \frac{r_{0 a}+\frac{1}{\kappa_{a}}(1-\cos (t))}{r_{a}}+\phi_{0 a}, \\
z_{a}=z_{0 a}+\gamma_{a} t
\end{gathered}
$$

where $\kappa_{a}$ is defined as the inverse of the radius of curvature (the sign determines the direction of the curvature), the vector $\left(r_{0 a}, \phi_{0 a}, z_{0 a}\right)$ defines the distance of the closest approach to the origin, and $\gamma_{a}[\mathrm{~m} / \mathrm{rad}]$ governs the translation along the $\mathrm{z}$-axis. This defines a semicircle which is sufficient to describe the particles of interest here.

The fact that the angle $\phi_{a}$ is not defined when $r_{a}=0$ is handled in appendix B.2.1.

From these equations it is possible to obtain the momentum and charge of the particles. The total momentum consists of two perpendicular components, the transverse and the longitudinal momentum. The ratio of the distances that are traversed in these to directions equals the ratio of these momenta.

$$
\frac{p_{t}}{p_{l}}=\frac{\frac{\Delta t}{\left|\kappa_{a}\right|}}{\Delta t\left|\gamma_{a}\right|}=\frac{1}{\left|\kappa_{a}\right|\left|\gamma_{a}\right|}
$$

Given equation 1 the longitudinal momentum is

$$
p_{l}=0.3 B\left|\gamma_{a}\right|
$$

and, hence

$$
\begin{aligned}
& p^{2}=p_{t}^{2}+p_{l}^{2}=(0.3 B)^{2}\left(\kappa_{a}^{-2}+\gamma_{a}^{2}\right) \quad\left[(\mathrm{GeV} / \mathrm{c})^{2}\right] \\
& q=-\operatorname{sgn}\left(\kappa_{a}\right)
\end{aligned}
$$

In order to obtain these parameters as accurately as possible, a fitting procedure must be used. What is wanted is to define the distance between each hit $\left(r_{i}, \phi_{i}, z_{i}\right)$ in the detector and each arm $a$. These distances are then to be minimized by manipulating the arm parameters. To be able to express a distance like this the position on the arm has to be obtained.

Again referring to figure 2, the angle $t$ for arm $a$, given a hit $\left(r_{i}, \phi_{i}\right)$ is

$$
\cos (t)=\frac{1+\kappa_{a} r_{0 a}-\kappa_{a} r_{i} \cos \left(\phi_{0 a}-\phi_{i}\right)}{\sqrt{\left(1+\kappa_{a} r_{0 a}-\kappa_{a} r_{i} \cos \left(\phi_{0 a}-\phi_{i}\right)\right)^{2}+\left(\kappa_{a} r_{i} \sin \left(\phi_{0 a}-\phi_{i}\right)\right)^{2}}}
$$

Eqs. 2-5 are all that is needed to proceed to define the distance between an arm and a hit, but one approximate parameterization is also given. 
If the helix begins in the vertex $r_{0 a}=0$, and for high energies $\cos (t) \approx 1-\frac{t^{2}}{2}$, the helix equations become

$$
\begin{gathered}
r_{a}=\frac{t}{\left|\kappa_{a}\right|} \\
\phi_{a}=\frac{\operatorname{sgn}\left(\kappa_{a}\right)}{2} t+\phi_{0 a}^{\prime} \\
z_{a}=z_{0 a}+\gamma_{a} t
\end{gathered}
$$

where $\phi_{0 a}^{\prime}$ is defined as

$$
\phi_{0 a}^{\prime}=\phi_{0 a}-\frac{\pi}{2}
$$

There are two ways to obtain the parameter $t$ : to use the expression in equation 5 which is difficult to simplify, or to define a distance $s$ in the $r-\phi$ plane according to

$$
s^{2}=\left(r_{a}-r_{i}\right)^{2}+\left(\phi_{a}-\phi_{i}\right)^{2}
$$

This is not a physical distance, but a way of using the fact that $r_{a} \propto \phi_{a}$ in this approximation. Differentiate $s$ with respect to $t$ and set it equal to zero to obtain the minimal distance.

$$
t=2\left|\kappa_{a}\right| \frac{2 r_{i}+\kappa_{a}\left(\phi_{i}-\phi_{0 a}^{\prime}\right)}{4+\kappa_{a}^{2}}
$$

The parameterization can of course be carried out in other ways, but for the ATLAS detector these two should be sufficient.

The squared perpendicular distance, $M_{i a}$, from arm $a$ to hit $i$ is, for any parameterization, given by

$$
\begin{aligned}
M_{i a} & =r_{a}^{2}+r_{i}^{2}-2 r_{a} r_{i} \cos \left(\phi_{a}-\phi_{i}\right)+\left(z_{a}-z_{i}\right)^{2}= \\
& =\left(r_{a}-r_{i}\right)^{2}+2 r_{a} r_{i}\left(1-\cos \left(\phi_{a}-\phi_{i}\right)\right)+\left(z_{a}-z_{i}\right)^{2}
\end{aligned}
$$

In this expression eqs. 2-5 or eqs. 6-9 are used to calculate $\left(r_{a}, \phi_{a}, z_{a}\right)$.

There is one detector problem left. In the barrel, one measurement error $(150 \mu \mathrm{m})$ is in the $r-\phi$ plane, and in the $z$ direction the error must be set equal to half the straw length $(47.5 \mathrm{~cm})$ since it is not known where along the straw the hit was made. In the end-cap there is a large error in the $r$ direction, and a small one in the $\phi-z$ plane. To make it possible to handle this an approximation is necessary.

$$
M_{i a} \approx\left(r_{a}-r_{i}\right)^{2}+2 r_{i}^{2}\left(1-\cos \left(\phi_{a}-\phi_{i}\right)\right)+\left(z_{a}-z_{i}\right)^{2}
$$


The approximation above is very accurate for small distances, the large distances are overestimated but these are anyway suppressed by the algorithm, as will be seen in sub-section 3.3, so this distance measure can be used.

It is now possible to rewrite $M_{i a}$ in the way that was suggested in [6] by normalizing the different coordinates with the error, much in the spirit of the standard chi-square fit. For notational simplicity $\sigma^{2}$ is replaced by $\rho$.

$$
\begin{aligned}
M_{i a} & =\frac{\left(r_{a}-r_{i}\right)^{2}}{\rho_{i}^{r}}+\frac{2 r_{i}^{2}\left(1-\cos \left(\phi_{a}-\phi_{i}\right)\right)}{\rho_{i}^{r \phi}}+\frac{\left(z_{a}-z_{i}\right)^{2}}{\rho_{i}^{z}}= \\
& =\frac{\left(r_{a}-r_{i}\right)^{2}}{\rho_{i}^{r}}+\frac{\left(1-\cos \left(\phi_{a}-\phi_{i}\right)\right)}{\frac{\rho_{i}^{r \phi} / r_{i}^{2}}{2}}+\frac{\left(z_{a}-z_{i}\right)^{2}}{\rho_{i}^{z}}= \\
& =\frac{M_{i a}^{r}}{\rho_{i}^{r}}+\frac{M_{i a}^{\phi}}{\rho_{i}^{\phi}}+\frac{M_{i a}^{z}}{\rho_{i}^{z}}
\end{aligned}
$$

Where the replacement

$$
\frac{\rho_{i}^{r \phi}}{2 r_{i}^{2}}=1-\left(1-\frac{\rho_{i}^{r \phi}}{2 r_{i}^{2}}\right) \approx 1-\cos \left(\frac{\sqrt{\rho_{i}^{r \phi}}}{r_{i}}\right) \equiv \rho_{i}^{\phi}
$$

has been made in the last step. The approximation is not necessary, but is made to elucidate the fact that this actually is the error in the $\phi$ term at a given radius $r_{i}$.

This new distance measure $M_{i a}$ is not a physical distance but the number of errors between $\operatorname{arm} a$ and hit $i$. In the barrel an $M_{i a}=1$ could mean $150 \mu m$ in the $r$ or $\phi$ directions, or $47.5 \mathrm{~cm}$ in the $z$ direction.

The results from this section will mainly come into use in section 3.3 , but some of them will be used in the Hough transform.

\subsection{Local Hough transform}

The local Hough transform is used to initiate the algorithm, that is to find the tracks and put an arm as close to the track as possible. The time consumption of the algorithm is proportional to the number of arms, there is a loop which goes over all arms, and therefore it is essential to give it a minimal number to work with. Still it is imperative to give it at least one arm per track because it cannot find a track without an arm to work with.

In the Hough transform the transverse momentum and azimuthal angle are calculated for every pair of two hits that are found in the detector. These values 
Figure 3: Trial event used throughout this work. All references which are made to one specific event refer to this.

are put into a histogram and any real track should form a significant peak. In [2] a local variant was used in which a circle is drawn around every hit and the calculation for the hits is only made within that circle, thereby decreasing the number of calculations that are needed. This can be limited further since the hits are given in increasing order of the radius, which makes it quite natural to just make the calculation for the succeeding hits and thereby limit the calculations to a circle sector outside every hit.

In [2] exact expressions to calculate the radius of curvature and azimuthal angle are used:

$$
\begin{aligned}
& \tan \phi_{0 a}^{\prime}=\frac{r_{j} \sin \phi_{i}-r_{i} \sin \phi_{j}}{r_{j} \cos \phi_{i}-r_{i} \cos \phi_{j}} \\
& \kappa_{a}=\frac{2}{r_{i}} \sin \left(\phi_{i}-\phi_{0 a}^{\prime}\right)
\end{aligned}
$$

But the trigonometric calculations needed are computationally demanding. If equation 6 and 7 are used instead, these expressions become

$$
\begin{gathered}
\phi_{0 a}^{\prime}=\frac{r_{j} \phi_{i}-r_{i} \phi_{j}}{r_{j}-r_{i}} \\
\kappa_{a}=\frac{2}{r_{i}}\left(\phi_{i}-\phi_{0 a}^{\prime}\right)
\end{gathered}
$$




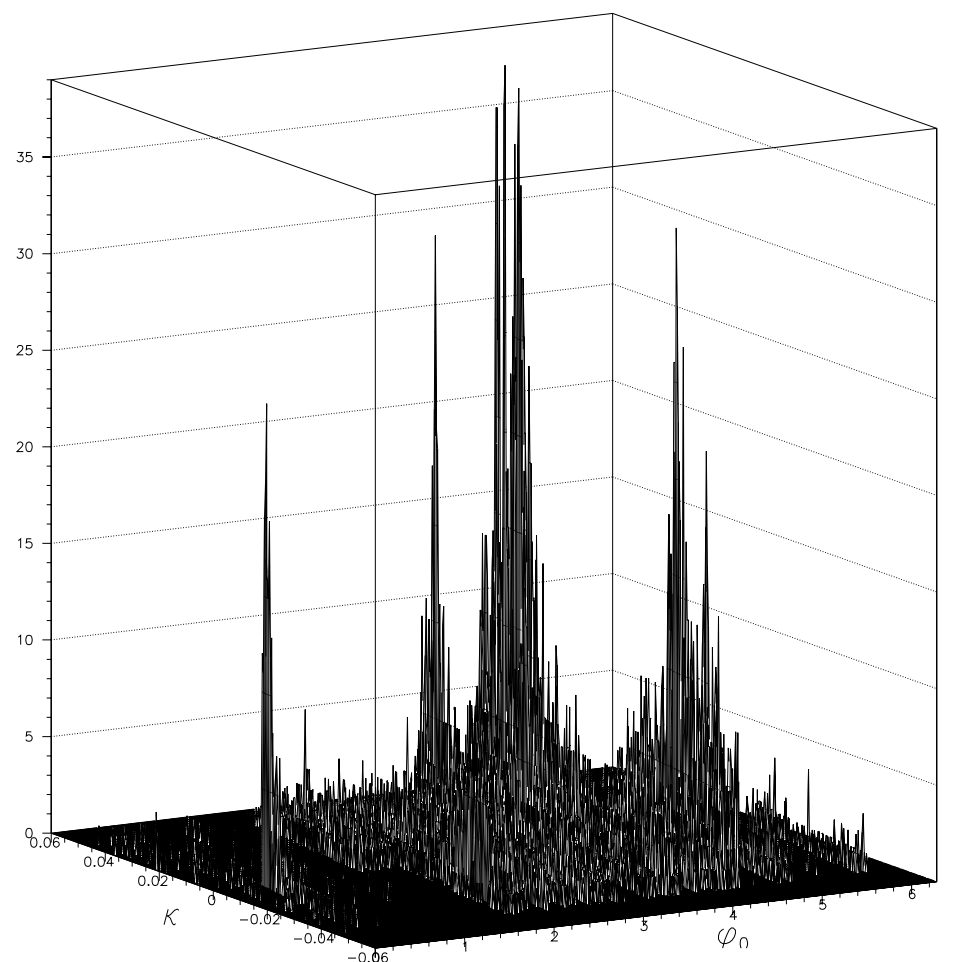

Figure 4: Hough-transform for the trial event. The histogram is 400 times 300 bins (see text)

and, thereby, the Hough transform becomes faster.

These values are put in a histogram, where the size of the histogram bins determines the resolution of the transform. As is given in [8] page 52, the error in measuring the transverse momentum of a charged particle in a magnetic field $B$ is given by

$$
\frac{\Delta p_{m}}{p}=\frac{\sigma_{x} p}{0.3 B L^{2}} \sqrt{\frac{720}{N+4}}
$$

where the measurement is performed by using $N$ equally spaced position measurements along $L$ meters of track, with $\sigma_{x}$ as the error in position measurement. This is used to calculate the size of the histogram bins.

$$
\left\{\begin{array}{l}
\frac{\Delta p_{t}}{p_{t n}^{2}}=k \\
\Delta p_{t}=p_{t n+1}-p_{t n}
\end{array}\right.
$$




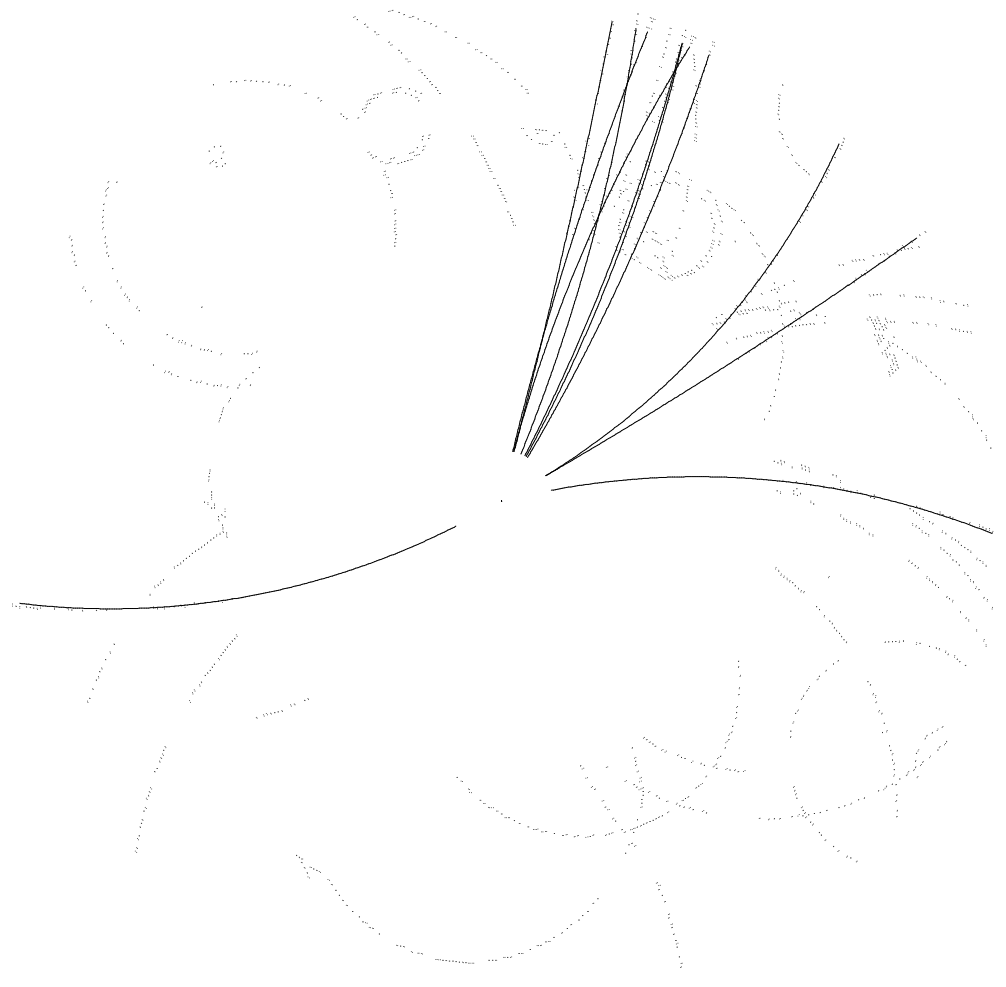

Figure 5: The trial event after the Hough transform.

$$
\Rightarrow p_{t n+1}=k p_{t n}^{2}+p_{t n}
$$

Equation 16 cannot be solved analytically, so an interval halving method is used to find the bin in which to put an entry. This might seem to be too much trouble but all approximations, linear and quadratic, have proven to be inadequate. They can be made to work in a limited momentum region, but all of them fail to find the tracks when the bin sizes are too small, alternatively it finds tracks with the wrong momentum if the bins are too big.

For $1<p_{t}<500 \mathrm{GeV} / \mathrm{c}$ and $k=0.01150$ bins are necessary in each $\kappa$ direction, and to match this with a corresponding resolution in $\phi, 400$ bins are required in that dimension. The resulting histogram for the trial event is shown in figure 4. The drift time is approximated here to be perpendicular to the radius, which for high energetic tracks is very accurate.

What is left is to sort out what peaks to use in the $\kappa-\phi$ histogram. The threshold must be chosen so that all tracks, whether they have left a large number of hits in the detector or not, are found and arms initiated close to them. It is impossible to achieve $100 \%$ efficiency but one must strive for it. On this basis the 
threshold for initiating an arm must be fairly low, which gives a large number of arms which really should not be there. This is sorted out in much the same way as in [2].

The initiated arms are taken one by one, and for every pair of hits, that are present within a certain distance from that arm, $\gamma_{a}$ and $z_{0 a}$ are calculated and put into a two dimensional histogram. In [2] the arms that had a substantial peak in this histogram went on to be treated by the fitting algorithm, but if, as in this case, a low threshold is chosen there will be a large number of initiated arms for most of the real tracks (i.e many hits in the detector makes them easy to locate) just because some are difficult to find. To eliminate the extra arms this part is done twice, the first time the height (only above a certain threshold) of the largest peak in the $\gamma_{a}, z_{0 a}$ histogram is saved, and after all arms have been treated they are sorted in decreasing order of their peaks.

In the second round those hits used (i.e within a short distance from the arm) are taken away so the subsequent arms cannot use them. This hit-arm distance must be very small (a straw diameter or so). A second selection is now made to pick those arms that are to be given to the algorithm. This has proved to be efficient in initiating one arm per track, close to their final positions.

\subsection{Elastic arm algorithm}

Given a set of arms and a set of hits in the detector, there are three problems to solve: two combinatorial and one continuous. First, which hits belong to a certain arm, next to determine which of the two drift time positions (the drift time is approximated to be perpendicular to the radius) should be used and finally what values the arm parameters should have when using the chosen hits. Normally these problems are solved one by one and a chi-square measure is used to determine whether it is a real track or not. If the track density is large this can be very difficult. That is if the average distance between two hits is too small, many mistakes can be made, both in the assignment and the fitting procedure. Hits can be 'stolen' from one arm and assigned to another, thereby making the fitting procedure very difficult. What is intended here is to incorporate the combinatorial problems into the continuous one. The remaining total problem is then solved with an iterative procedure for all arms at the same time. The basic principle derived in $[2]$ and $[6]$ is to form an error measure:

$$
E\left(\left\{S_{i a}, s_{i a}^{ \pm}\right\} ; \vec{\pi}\right)=\sum_{i}\left(\sum_{a} S_{i a}\left(s_{i a}^{+} M_{i a}^{+}+s_{i a}^{-} M_{i a}^{-}\right)+\lambda\left(\sum_{a} S_{i a}-1\right)^{2}\right)
$$

where $i=1, . ., n$ denotes the hits and $a=1, . ., m$ denotes the arms. The binary decision units $S_{i a}$ are defined as $S_{i a}=1$ if hit $i$ belongs to arm $a$ and zero otherwise. Since there was only one particle that made a hit, $S_{i a}$ can only be equal to 1 for one arm for each hit. If $S_{i a}=0$ for all arms, the hit is assigned to noise and is given the weight $\lambda$. Furthermore, the ambiguity units $s_{i a}^{ \pm}$are defined 
as $s_{i a}^{+}+s_{i a}^{-}=1 \forall i, a$. That is the arm belongs to one and only one of the two possible positions that are defined by the drift time.

The notation $\vec{\pi}$ stands for one set of parameters $\left(\kappa_{a}, r_{0 a}, \phi_{0 a}, \gamma_{a}, z_{0 a}\right)$ per arm a. The ideal parameters are of course when the arm is going through all the hits that have been assigned to it by $\left\{S_{i a}, s_{i a}^{ \pm}\right\}$. This will give a zero error.

The error measure contains all the information needed to make the reconstruction. For every assignment it is possible to minimize the error measure by manipulating the parameters, but the different assignment sets are still separated. As a first step in adding them to the continuous problem, noise is added to the error measures. This is done with the Boltzmann distribution.

$$
P\left(\left\{S_{i a}, s_{i a}^{ \pm}\right\} ; \vec{\pi}\right)=\frac{e^{-\beta E\left(\left\{S_{i a}, s_{i a}^{ \pm}\right\} ; \vec{\pi}\right)}}{Z}
$$

where $Z$ is the partition function and $\beta$ the inverse temperature

$$
\begin{aligned}
Z & =\sum_{\left\{S_{i a}, s_{i a}^{ \pm}\right\}} \int d^{n} \vec{\pi} e^{-\beta E\left(\left\{S_{i a}, s_{i a}^{ \pm}\right\} ; \vec{\pi}\right)} \\
\beta & \equiv \frac{1}{T}
\end{aligned}
$$

This 'temperature' is not related to the physical temperature, but is a control parameter governing the width of the distribution.

The probability shall be maximized, because the configuration that has the least error according to equation 17 has the largest probability. In appendix A it is shown that there exists $(2+2 m)^{n}$ sets of 'legal' combinations of $\left\{S_{i a}, s_{i a}^{ \pm}\right\}$. To try to find the most probable combination of these by a trial and error method is not feasible, so a different approach is used.

The 'good' combinations will have a much larger probability than the totally irrelevant ones (take a couple of hits which form a zigzag pattern and assign to an arm will result in a large error measure). If the distributions of all combinations are added together and viewed as one, then the 'good' combinations will dominate the distribution at low temperatures. This sum is a marginal probability distribution since it is only a sum of the assignment combinations; the arm parameters are left as free variables.

$$
P_{M}(\vec{\pi})=\sum_{\left\{S_{i a}, s_{i a}^{ \pm}\right\}} P\left(\left\{S_{i a}, s_{i a}^{ \pm}\right\} ; \vec{\pi}\right)
$$

It is possible to rewrite this (which is done in appendix A) to one probability distribution which we then want to maximize. 


$$
P_{M}(\vec{\pi})=\frac{e^{-\beta E_{e f f}}}{Z}
$$

Maximizing a distribution like this is the same as minimizing its exponent. In this case the exponent is denoted as the effective energy.

$$
E_{e f f}=-\frac{1}{\beta} \sum_{i} \ln \left(2 e^{-\beta \lambda}+\sum_{a}\left(e^{-\beta M_{i a}^{+}}+e^{-\beta M_{i a}^{-}}\right)\right)
$$

The effective energy, $E_{\text {eff }}$, contains all three problems. The two combinatorial problems have been reduced to local minima in an energy landscape, so what we want to obtain are the values of the arm parameters in the global minimum. To find this the gradient descent method is used, which uses the gradient to point out the direction of the steepest slope.

$$
\begin{aligned}
& \Delta \kappa_{a}=-\eta \frac{\partial E_{e f f}}{\partial \kappa_{a}}=-\eta \sum_{i} V_{i a}\left(v_{i a}^{+} \frac{\partial M_{i a}^{+}}{\partial \kappa_{a}}+v_{i a}^{-} \frac{\partial M_{i a}^{-}}{\partial \kappa_{a}}\right) \\
& \Delta r_{0 a}=-\eta \frac{\partial E_{e f f}}{\partial r_{0 a}}=-\eta \sum_{i} V_{i a}\left(v_{i a}^{+} \frac{\partial M_{i a}^{+}}{\partial r_{0 a}}+v_{i a}^{-} \frac{\partial M_{i a}^{-}}{\partial r_{0 a}}\right) \\
& \Delta \phi_{0 a}=-\eta \frac{\partial E_{e f f}}{\partial \phi_{0 a}}=-\eta \sum_{i} V_{i a}\left(v_{i a}^{+} \frac{\partial M_{i a}^{+}}{\partial \phi_{0 a}}+v_{i a}^{-} \frac{\partial M_{i a}^{-}}{\partial \phi_{0 a}}\right) \\
& \Delta \gamma_{a}=-\eta \frac{\partial E_{e f f}}{\partial \gamma_{a}}=-\eta \sum_{i} V_{i a}\left(v_{i a}^{+} \frac{\partial M_{i a}^{+}}{\partial \gamma_{a}}+v_{i a}^{-} \frac{\partial M_{i a}^{-}}{\partial \gamma_{a}}\right) \\
& \Delta z_{0 a}=-\eta \frac{\partial E_{e f f}}{\partial z_{0 a}}=-\eta \sum_{i} V_{i a}\left(v_{i a}^{+} \frac{\partial M_{i a}^{+}}{\partial z_{0 a}}+v_{i a}^{-} \frac{\partial M_{i a}^{-}}{\partial z_{0 a}}\right)
\end{aligned}
$$

where

$$
\begin{gathered}
V_{i a}=\frac{e^{-\beta M_{i a}^{+}}+e^{-\beta M_{i a}^{-}}}{2 e^{-\beta \lambda}+\sum_{b}\left(e^{-\beta M_{i b}^{+}}+e^{-\beta M_{i b}^{-}}\right)} \\
v_{i a}^{ \pm}=\frac{e^{-\beta M_{i a}^{+}}}{e^{-\beta M_{i a}^{+}}+e^{-\beta M_{i a}^{-}}}
\end{gathered}
$$

The assignment probabilities $\left(V_{i a}, v_{i a}^{ \pm}\right)$correspond to the original binary decision units $S_{i a}, s_{i a}^{ \pm}$in a natural way. This in the sense that the $V_{i a}$ 's decide by probability which hits will be assigned to a track and the $v_{i a}^{ \pm}$'s decides which in the pair will be assigned to the track. Strictly speaking they are the temperature 
average of the binary decision units $V_{i a}=\left\langle S_{i a}\right\rangle_{\beta}$. All this is described in [2] and [4].

The system is now started at a reasonably low temperature, high enough to incorporate the good assignment sets and low enough to exclude the bad ones. We then try to find the optimal set of parameters at this temperature, thereafter we lower the temperature one small step and do the optimization again. Eventually we will find the global minimum. This procedure is called deterministic annealing. It is closely related to simulated annealing, this expression being taken from the experimental world because of the direct analogy to freezing down a specimen. Some basic information about this can be studied in $[10]$ page $25-35$ and page $72-76$.

The update rate $\eta$, the speed at which we slide down the slope, is the next problem and several different solutions can be used.

\subsubsection{Hessian matrix}

It still remains to estimate how large steps $\eta$ to take. This might seem an easy task but there are a variety of ways to do it. In [2] M.Ohlsson tried to set them to fixed values and in [4] he used the metric tensor to decide the update rates. In [4] he also pointed out that the so-called Hessian matrix could be used. It consists of the second derivatives and can be understood by the Taylor expansion of $E_{e f f}$ at a point $p$ in parameter space.

$$
E_{e f f}(\vec{\pi}) \approx E_{e f f}\left(\overrightarrow{\pi_{p}}\right)+\left(\left.\nabla E_{e f f}\right|_{\overrightarrow{\pi_{p}}}\right) \cdot \Delta \pi+\frac{1}{2} \Delta \pi \cdot\left(\left.H\right|_{\overrightarrow{\pi_{p}}}\right) \cdot \Delta \pi
$$

where the Hessian matrix is

$$
H=\left(\begin{array}{ccccc}
\frac{\partial^{2} E_{e f f}}{\partial \kappa_{a}^{2}} & \frac{\partial^{2} E_{e f f}}{\partial r_{0 a} \partial \kappa_{a}} & \frac{\partial^{2} E_{e f f}}{\partial \phi_{0 a} \partial \kappa_{a}} & \frac{\partial^{2} E_{e f f}}{\partial \gamma_{a} \partial \kappa_{a}} & \frac{\partial^{2} E_{e f f}}{\partial z_{0 a} \partial \kappa_{a}} \\
\frac{\partial^{2} E_{e f f}}{\partial \kappa_{a} \partial r_{0 a}} & \frac{\partial^{2} E_{e f f}}{\partial r_{0 a}^{2}} & \frac{\partial^{2} E_{e f f}}{\partial \phi_{0 a} \partial r_{0 a}} & \frac{\partial^{2} E_{e f f}}{\partial \gamma_{a} \partial r_{0 a}} & \frac{\partial^{2} E_{e f f}}{\partial z_{0 a} \partial r_{0 a}} \\
\frac{\partial^{2} E_{e f f}}{\partial \kappa_{a} \partial \phi_{0 a}} & \frac{\partial^{2} E_{e f f}}{\partial r_{0 a} \partial \phi_{0 a}} & \frac{\partial^{2} E_{e f f}}{\partial \phi_{0 a}^{2}} & \frac{\partial^{2} E_{e f f}}{\partial \gamma_{a} \partial \phi_{0 a}} & \frac{\partial^{2} E_{e f f}}{\partial z_{0 a} \partial \phi_{0 a}} \\
\frac{\partial^{2} E_{e f f}}{\partial \kappa_{a} \partial \gamma_{a}} & \frac{\partial^{2} E_{e f f}}{\partial r_{0 a} \partial \gamma_{a}} & \frac{\partial^{2} E_{e f f}}{\partial \phi_{0 a} \partial \gamma_{a}} & \frac{\partial^{2} E_{e f f}}{\partial \gamma_{a}^{2}} & \frac{\partial^{2} E_{e f f}}{\partial z_{0 a} \partial \gamma_{a}} \\
\frac{\partial^{2} E_{e f f}}{\partial \kappa_{a} \partial z_{0 a}} & \frac{\partial^{2} E_{e f f}}{\partial r_{0 a} \partial z_{0 a}} & \frac{\partial^{2} E_{e f f}}{\partial \phi_{0 a} \partial z_{0 a}} & \frac{\partial^{2} E_{e f f}}{\partial \gamma_{a} \partial z_{0 a}} & \frac{\partial^{2} E_{e f f}}{\partial z_{0 a}^{2}}
\end{array}\right)
$$

If the point which is reached with a movement $\Delta \pi$ is the global minimum then the gradient of equation 25 will vanish.

$$
\nabla E_{e f f}(\vec{\pi})=\left(\left.\nabla E_{e f f}\right|_{\pi_{p}}\right)+\left(\left.H\right|_{\pi_{p}}\right) \cdot \Delta \pi=0
$$


Both the gradient and the Hessian matrix can be computed. The inverse matrix gives the updating rule

$$
\Delta \pi_{a}=-\epsilon H_{a}^{-1} \cdot \nabla_{\pi_{a}} E_{e f f}
$$

where $\epsilon$ is the global update rate which can be used to slow down or speed up the process. The first derivatives are given by equations 20-24. The index $a$ in equation 26 denotes that this has to be calculated for all arms. The calculation of the Hessian is not complicated but it gives quite long expressions for the exact parameterizations. This is done in appendix B for both parameterizations, so this section is concluded by certifying that it can be done without any great loss of time and that the results are very rewarding.

\subsection{Program}

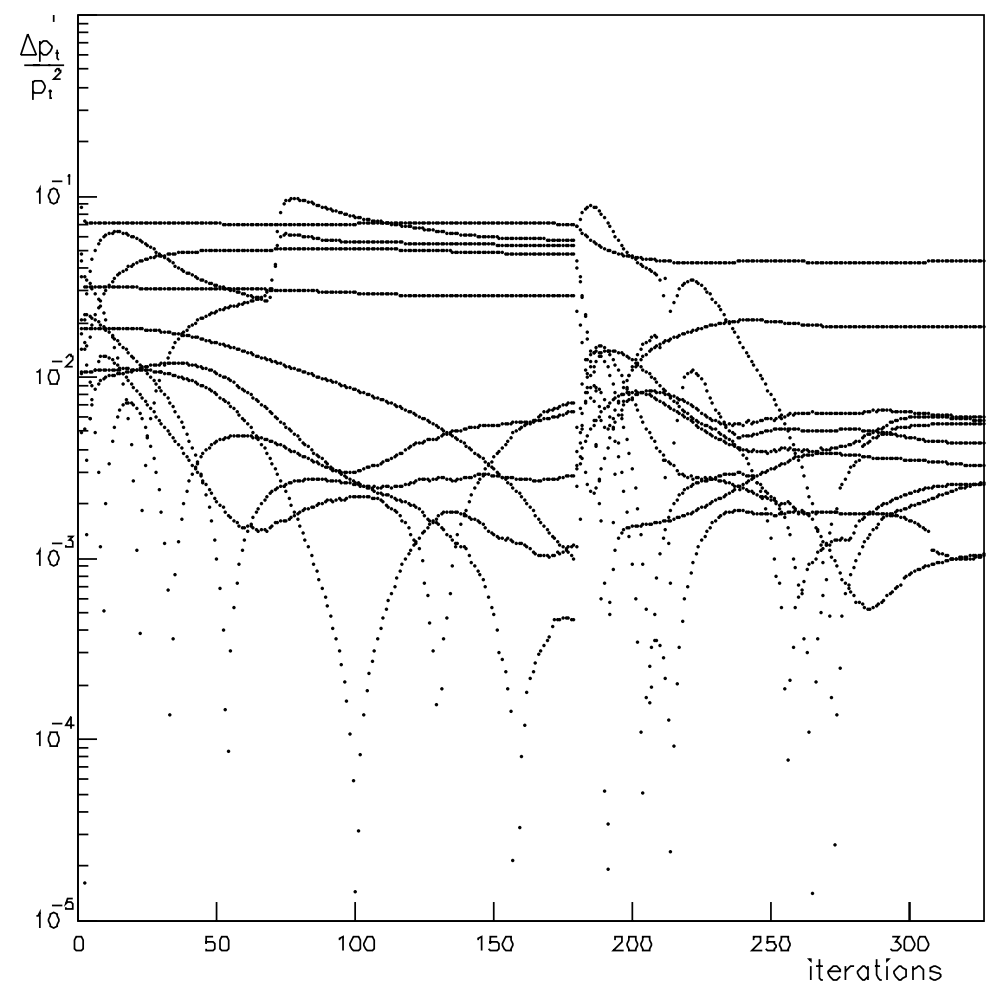

Figure 6: Convergence of the trial event. $\frac{\Delta p_{t}}{p_{t}^{2}}$ as function of the iterations for all 11 arms. The approximate fit on the left is 187 iterations long.

A description of the program that was used follows 
- Hough transform.

1. Loop over all hits and calculate $\kappa$ and $\phi_{0}^{\prime}$ according to eq. 13- 14, and put them in a histogram.

2. Initiate arms with the parameter values corresponding to the highest peaks.

3. Loop over all initiated arms and calculate $\gamma$ and $z_{0}$ for the hits that reside within a small distance from the arm, and put into one histogram per arm.

4. Save the height of the highest peak for every arm.

5. Sort the arms in decreasing order of those peaks.

6. Repeat item 3 but remove the used hits so the succeeding arms cannot use them.

7. Remove those arms that get too few hits in the second round, transfer the remaining ones to the algorithm.

- The algorithm.

1. Calculate the effective energy (loop over all hits and all arms).

2. Loop over all arms

(a) Calculate the first and second derivatives of the effective energy for the chosen parameterization (loop over all hits).

(b) Update the parameters according to $\pi=\pi+\Delta \pi$, where $\Delta \pi$ is given by eq. 26 .

(c) Change the assignment probabilities according to the new parameters (loop over all hits).

3. Lower the temperature and start again from item 1, until the final temperature is reached.

The starting temperature was set to 200 , and the final to 0.5 for the approximate parameterization and $\mathbf{0 . 0 5}$ for the exact. It was decreased according to the rule

$$
T_{\text {new }}=0.95 \cdot T_{\text {old }}
$$

The extra loop in the algorithms item $2 \mathrm{c}$ is needed if two tracks are very close to each other. At a high temperature they will form one minimum into which both arms will fall. As the annealing proceeds, that minimum will split into two. If both arms were allowed to go to the deepest minimum (the track that has the most hits) the other would be missed. By changing the assignment probabilities after the first arm has started to move towards its minimum, the other is not 
prohibited to go there, but it will most likely go to the other minimum where all hits are free to be used.

For the exact parameterization a 'thermalizing' process, using a low update rate $\epsilon=0.05$ initially, was necessary to keep it from jumping out from the vertex. The update rate was increased during 10 iterations until it reached unity.

The result for the trial event is shown in figure 6 , iteration by iteration. It starts from the left with the values given by the Hough transform. The algorithm tries to adjust the arm parameters to place them in the minima, and the 'dips' in error are when it passes the correct, simulated, $p_{t}$.

\section{Results}

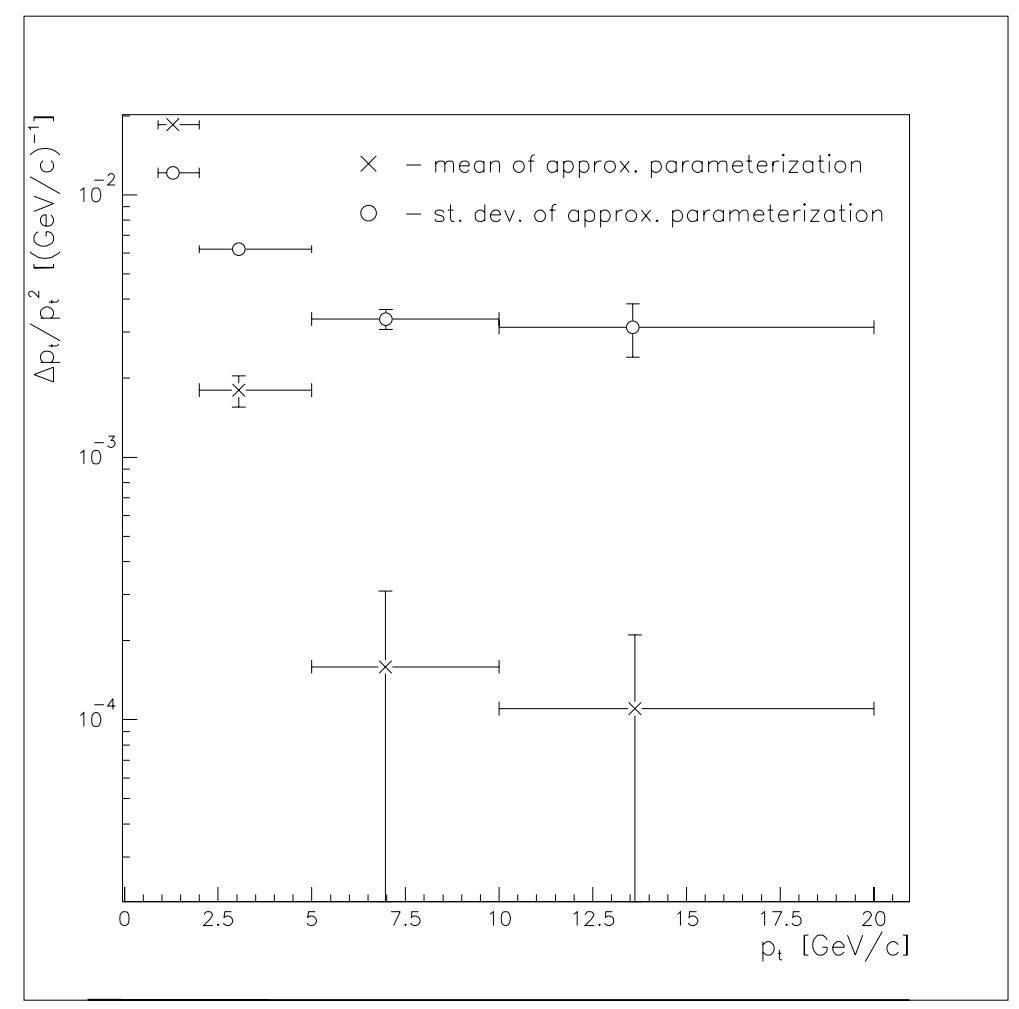

Figure 7: Bias of the approximate parameterization, with 1779 tracks in 220 events. The mean $\frac{\Delta p_{t}}{p_{t}^{2}}$ in the interval is marked with a cross, and the standard deviation of $\frac{\Delta p_{t}}{p_{t}^{2}}$ of the reconstructed tracks with error bars is marked with a circle.

The efficiency depends on the number of tracks the Hough transform finds. The program used here has found $85 \%$ of the tracks above $1 \mathrm{GeV} / \mathrm{c}$ that pass through 


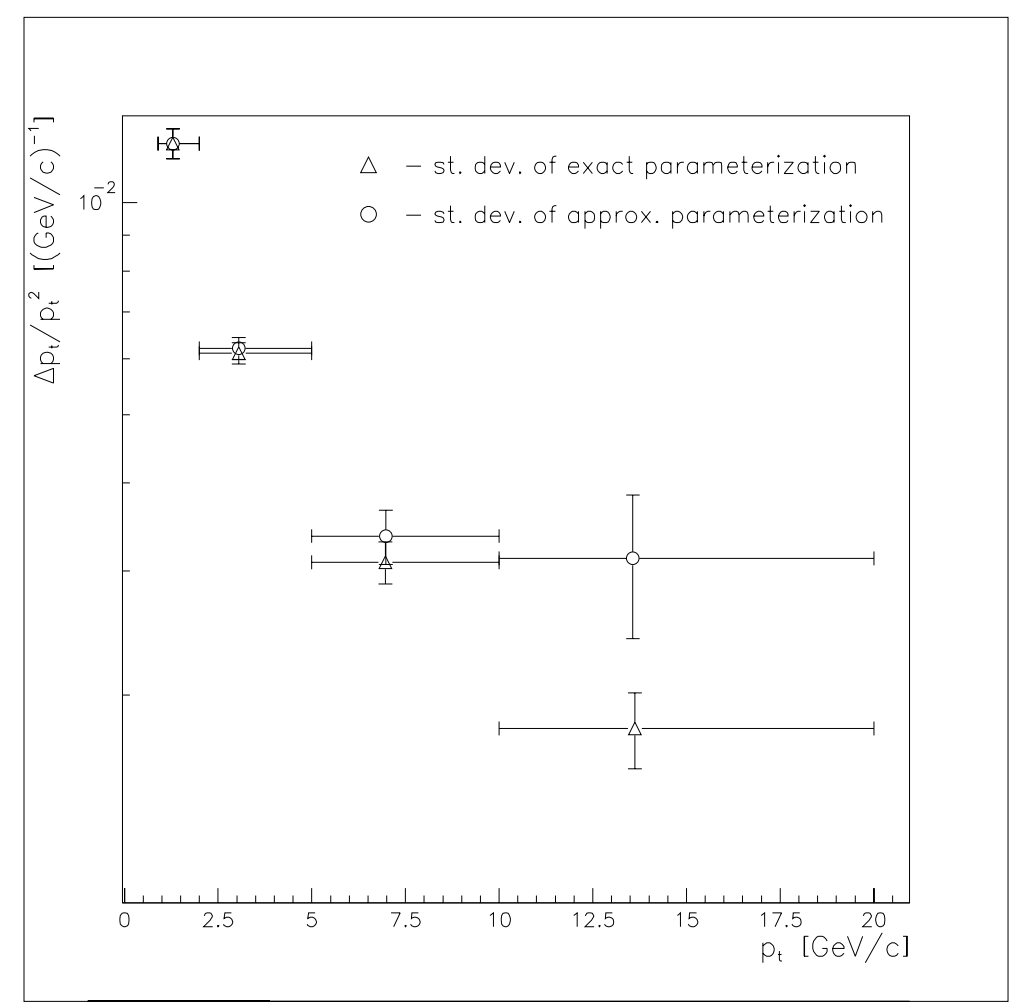

Figure 8: Results from the algorithm, with 1779 tracks in 220 events. The standard deviation of $\frac{\Delta p_{t}}{p_{t}^{2}}$ of the reconstructed tracks with error bars are marked.

the barrel from the inner horizontal surface to the outer.

The low efficiency comes from the fact that the calculations in the Hough transform are made under the assumption that $r_{0}=0$ with the use of the approximate equations. This smears out the high energy tracks. These smeared out tracks will be covered by noise because the calculations had to be made for both ambiguity positions, which of course gave an increase in the number of entries in the histogram. The natural way to improve efficiency would be to increase the bin size in the histogram to collect the track in one bin, but this would lead to a poorer resolution and tracks would be missed because of this. The best solution would be to find a way of avoiding the double calculation because of the ambiguity, and thereby reducing the noise so the present resolution could be kept. For further understanding of the smearing out of the high energy tracks, the results from the approximate parameterizations in figure 7 and the discussion further on in this section should be studied.

For those tracks that only partially pass through, i.e. going in or out of the detector through the vertical sides, the efficiency is $65 \%$. These tracks leave fewer hits and are thus more difficult to locate. 
The results after 220 jet events are still very encouraging. The algorithm gives good results for the exact equations. In figure 8 the standard deviation of the reconstructed tracks $\frac{\Delta p_{t}}{p_{t}^{2}}$ is displayed and as expected it is larger for the low momenta because of multiple scattering. For the high momentum results it is possible to compare them to the theoretical values which can be calculated using equation 15. For this we need to know how many hits are associated with the arm in question.

After the algorithm has converged it is quite easy to sort out which arms that have found true tracks with the use of the sum of assignment probabilities

$$
N(a)=\sum_{i} V_{i a}
$$

which, in the low temperature limit, gives a measure of the number of hits that are assigned to a track. Here the $V_{i a}$ 's are almost one or zero. Therefore in the sum in eq. 27 , a contribution of one is given for the hits that are used and zero for the rest.

The average $N(a)$ for the reconstructed tracks with $10<p_{t}<20 \mathrm{GeV} / \mathrm{c}$ is 29 and using equation 15 , with $\sigma_{x}=150 \mu m$ and $L=0.95 m$, the error is $1.29 \cdot 10^{-3}(\mathrm{GeV} / \mathrm{c})^{-1}$. This is comparable to the result in figure 8 which is 1.79 . $10^{-3}(\mathrm{GeV} / \mathrm{c})^{-1}$.

For the approximate parameterization, the average $\frac{\Delta p_{t}}{p_{t}^{2}}$ for the low momentum arms is displaced from zero because the arms do not attach themselves to the hits as desired. The numerator in equation 6 will be too big, so the radial coordinate will be too large and the arm will lie outside the ideal placement. This has the effect that the algorithm is increasing $\kappa$ to compensate for this and thereby gives the arm a lower momentum than required.

In the high momentum region the fact that $r_{0}=0$ is important. For these more or less straight tracks a small displacement in the vertex is vital, the arms cannot connect to the hits.

As can be seen in figure 7 , the error in the determination of the standard deviation is increasing for high momentum tracks. The distribution of the $\frac{\Delta p_{t}}{p_{t}^{2}}$ 's should be gaussian, centered around zero, but in this case it was difficult to get the distribution to fit. The improvement that can be made is to add a correction for $r_{0}$ which should help in the high momentum region.

Referring to the table below, it is quite obvious that the approximate parameterizations should be used. The complexity of the exact equations makes the computations demanding, and the approximate solutions give accurate enough results.

CPU consumption on HP 735

\begin{tabular}{|c|c|c|}
\hline Hough & Approx. & Exact \\
\hline $\mathcal{O}(5 \mathrm{~s})$. & $\mathcal{O}(20 \mathrm{~s})$. & $\mathcal{O}(60 \mathrm{~s})$. \\
\hline
\end{tabular}


To make the algorithm work properly the efficiency must be improved. Some way of avoiding the double ambiguity calculation should be investigated. To improve the final results a new approximate parameterization which takes $r_{0}$ in consideration should be derived and applied. Furthermore, the range of the gaussian distributions around the arms should be considered when optimizing the program. It is not necessary to loop over all hits for all arms since the assignment probabilities decreases rapidly with the distance. It is of course enough to make the summation of those hits that make a numerical contribution to the update equations for a certain arm. However, one must be careful when implementing such optimization since the arms can move in the detector and thereby make changes in the assignment probabilities. These improvements will make the algorithm work both efficiently and fast.

\section{Acknowledgments}

I wish to thank Ulf Mjörnmark for his assistance with the simulations and in the proper use of the computers, Mattias Ohlsson for help with the algorithm, and also my supervisor Torsten Åkesson.

\section{A Derivation of the algorithm}

This derivation is an expanded variant of what M.Ohlsson has done in [2] and [9] where I have incorporated the treatment of ambiguities that were suggested by R.Blankenbecler in [6]. We start from the error measure

$$
E\left(\left\{S_{i a}, s_{i a}^{ \pm}\right\} ; \vec{\pi}\right)=\sum_{i}\left(\sum_{a} S_{i a}\left(s_{i a}^{+} M_{i a}^{+}+s_{i a}^{-} M_{i a}^{-}\right)+\lambda\left(\sum_{a} S_{i a}-1\right)^{2}\right)
$$

where $i=1, . ., n$ and $a=1, . ., m$. The binary decision units $S_{i a}$ are defined as $S_{i a}=1$ if hit $i$ belongs to track $a$ and zero otherwise. Furthermore, the ambiguity units $s_{i a}^{ \pm}$are defined as $s_{i a}^{+}+s_{i a}^{-}=1 \forall i, a$. The constraints

$$
\begin{aligned}
& \sigma(i) \equiv \sum_{a} S_{i a}=\left\{\begin{array}{l}
1 \text { hit } \\
0 \text { noise }
\end{array} \forall i\right. \\
& \sigma^{+}(i) \equiv \sum_{a} s_{i a}^{+}=\left\{\begin{array}{l}
m \text { if hit } i^{+} \text {is the true signal } \\
\left.0 \text { otherwise (i.e. assignment to } i^{-}\right)
\end{array} \forall i\right. \\
& \sigma^{-}(i) \equiv \sum_{a} s_{i a}^{-}=\left\{\begin{array}{l}
m \text { if hit } i^{-} \text {is the true signal } \\
\left.0 \text { otherwise (i.e. assignment to } i^{+}\right)
\end{array} \forall i\right.
\end{aligned}
$$

states that a hit can belong to one and only one track alternatively be assigned to the noise parameter $\lambda$. Furthermore, a track can only be assigned to one of 
the two ambiguities $s_{i a}^{+}$and $s_{i a}^{-}$.

To incorporate all problems into one function that can be minimized, we start by adding noise in the form of the Boltzmann distribution.

$P\left(\left\{S_{i a}, s_{i a}^{ \pm}\right\} ; \vec{\pi}\right)=\frac{e^{-\beta E\left(\left\{S_{i a}, s_{i a}^{ \pm}\right\} ; \vec{\pi}\right)}}{Z}$

where $Z$ is the partition function

$Z=\sum_{\left\{S_{i a}, s_{i a}^{ \pm}\right\}} \int d^{n} \vec{\pi} e^{-\beta E\left(\left\{S_{i a}, s_{i a}^{ \pm}\right\} ; \vec{\pi}\right)}$

The next step is to calculate the marginal probability

$P_{M}(\vec{\pi})=\sum_{\left\{S_{i a}, s_{i a}^{ \pm}\right\}} P\left(\left\{S_{i a}, s_{i a}^{ \pm}\right\} ; \vec{\pi}\right)$

where the sum is over all sets of $S_{i a}, s_{i a}^{ \pm}$'s which satisfies the global constraints above. The question to answer is how many such sets there are.

Summation table for the neural decision units

\begin{tabular}{c|c|c|c|c||c} 
& $S_{i 1}, s_{i 1}^{ \pm}$ & $S_{i 2}, s_{i 2}^{ \pm}$ & $\ldots$ & $S_{i m}, s_{i m}^{ \pm}$ & $\sigma(i), \sigma^{ \pm}(i)$ \\
\hline$S_{1 a}, s_{1 a}^{ \pm}$ & $1 / 0$ & $1 / 0$ & & $1 / 0$ & $1 / 0$ \\
\hline$S_{2 a}, s_{2 a}^{ \pm}$ & $1 / 0$ & $1 / 0$ & & $1 / 0$ & $1 / 0$ \\
\hline$\vdots$ & & & & & \\
\hline$S_{n a}, s_{n a}^{ \pm}$ & $1 / 0$ & $1 / 0$ & & $1 / 0$ & $1 / 0$
\end{tabular}

With the help of the table it can be seen that if

$\sigma(i)=1 \forall i \Rightarrow(2 m)^{n}$ such sets

and because of the ambiguity terms every noise hit can also be chosen in two different ways so

$\sigma(i)=1 \forall i$ except one $\Rightarrow 2 n(2 m)^{n-1}$ such sets

etc. This gives the total number of sets as

$$
\begin{aligned}
& S=(2 m)^{n}+n(2 m)^{n-1} 2+\left(\begin{array}{c}
n \\
2
\end{array}\right)(2 m)^{n-2} 2^{2}+\ldots+\left(\begin{array}{c}
n \\
n-1
\end{array}\right)(2 m) 2^{n-1}+2^{n}= \\
& =\sum_{k=0}^{n}\left(\begin{array}{c}
n \\
k
\end{array}\right)(2 m)^{n-k} 2^{k}=(2 m+2)^{n}
\end{aligned}
$$

The next step is to define a function $\alpha(i)$ so that if $\sigma(i)=1$ then $S_{i \alpha(i)}=1$ for any set $\left\{S_{i a}, s_{i a}^{ \pm}\right\}$which satisfies the global constraints. It gives the arm number to 
which hit $i$ is assigned. If it is assigned to the noise parameter $\lambda$ we set $\alpha(i)=0$. We then use this to rewrite the marginal probability as

$$
\begin{aligned}
Z P_{M} & =\sum_{\alpha(i)} e^{-\beta \sum_{i}\left(\delta_{\sigma(i), 1}\left(M_{i a}^{+} \delta_{\sigma+(i), 1}+M_{i a}^{-} \delta_{\sigma-(i), 1}\right)+\lambda \delta_{\sigma(i), 0}\right)}= \\
& =\sum_{\alpha(i)} \prod_{i} e^{-\beta\left(\delta_{\sigma(i), 1}\left(M_{i a}^{+} \delta_{\sigma+(i), 1}+M_{i a}^{-} \delta_{\sigma-(i), 1}\right)+\lambda \delta_{\sigma(i), 0}\right)}
\end{aligned}
$$

This sum of $(2 m+2)^{n}$ products can be rewritten as a product of $(2 m+2)$ factors as

$$
Z P_{M}=\prod_{i}\left(2 e^{-\beta \lambda}+\sum_{a}\left(e^{-\beta M_{i a}^{+}}+e^{-\beta M_{i a}^{-}}\right)\right)=e^{-\beta E_{e f f}}
$$

where

$$
E_{e f f}=-\frac{1}{\beta} \sum_{i} \ln \left(2 e^{-\beta \lambda}+\sum_{a}\left(e^{-\beta M_{i a}^{+}}+e^{-\beta M_{i a}^{-}}\right)\right)
$$

The solution to the track finding problem is in $E_{\text {eff }}$ 's global minimum, and the procedure to find this is derived in section 3.3.

\section{B Gradient descent equations and Hessian ma- trix}

The equations corresponding to the minimizing procedure derived in section 3.3 and 3.3.1 are derived here for the two different parameterizations which were given in section 3.1 .

What is needed is to calculate the first and second derivatives of the effective energy (equation 19).

$E_{e f f}=-\frac{1}{\beta} \sum_{i} \ln \left(2 e^{-\beta \lambda}+\sum_{a}\left(e^{-\beta M_{i a}^{+}}+e^{-\beta M_{i a}^{-}}\right)\right)$

The first derivatives are given by eqs. 20-24 in section 3.3. From these it is possible to calculate the gradient descent equations. They contain the derivatives of $M_{i a}^{ \pm}$and it remains to calculate these, but the first step to obtain the Hessian matrix is taken first.

With the notation $\pi=\left\{\kappa_{a}, r_{0 a}, \phi_{0 a}, \gamma_{a}, z_{0 a}\right\}$ the second derivatives of the effective energy become

$$
\frac{\partial^{2} E_{e f f}}{\partial \pi_{k} \partial \pi_{l}}=\sum_{i} V_{i a}\left(v_{i a}^{+}\left(-\beta\left(1-V_{i a} v_{i a}^{+}\right) \frac{\partial M_{i a}^{+}}{\partial \pi_{k}} \frac{\partial M_{i a}^{+}}{\partial \pi_{l}}+\frac{\partial^{2} M_{i a}^{+}}{\partial \pi_{k} \partial \pi_{l}}\right)+\right.
$$




$$
\begin{aligned}
+v_{i a}^{-} & \left.-\beta\left(1-V_{i a} v_{i a}^{-}\right) \frac{\partial M_{i a}^{-}}{\partial \pi_{k}} \frac{\partial M_{i a}^{-}}{\partial \pi_{l}}+\frac{\partial^{2} M_{i a}^{-}}{\partial \pi_{k} \partial \pi_{l}}\right)- \\
- & \left.V_{i a} v_{i a}^{+} v_{i a}^{-}\left(\frac{\partial M_{i a}^{+}}{\partial \pi_{k}} \frac{\partial M_{i a}^{-}}{\partial \pi_{l}}+\frac{\partial M_{i a}^{-}}{\partial \pi_{k}} \frac{\partial M_{i a}^{+}}{\partial \pi_{l}}\right)\right)
\end{aligned}
$$

These expressions are the contributions to the elements of the Hessian matrix and as can be seen they contain the derivatives of $M_{i a}$ with respect to the track parameters. The matrix and the gradient descent equations have several common factors which only have to be calculated once and then simply put into the final expressions. By formulating a tree structure where the used values can be extracted and saved, to finally being used in the formation of the update equation, an efficient structure is obtained.

Upon differentiating equation 11 once one obtains

$$
\begin{aligned}
\frac{\partial M_{i a}^{r}}{\partial \pi_{l}} & =\frac{2\left(r_{a}-r_{i}\right)}{\rho_{i}^{r}} \frac{\partial r_{a}}{\partial \pi_{l}} \\
\frac{\partial M_{i a}^{\phi}}{\partial \pi_{l}} & =\frac{\sin \left(\phi_{a}-\phi_{i}\right)}{\rho_{i}^{\phi}} \frac{\partial \phi_{a}}{\partial \pi_{l}} \\
\frac{\partial M_{i a}^{z}}{\partial \pi_{l}} & =\frac{2\left(z_{a}-z_{i}\right)}{\rho_{i}^{z}} \frac{\partial z_{a}}{\partial \pi_{l}}
\end{aligned}
$$

and twice

$$
\begin{aligned}
\frac{\partial^{2} M_{i a}^{r}}{\partial \pi_{k} \partial \pi_{l}} & =\frac{2}{\rho_{i}^{r}} \frac{\partial r_{a}}{\partial \pi_{k}} \frac{\partial r_{a}}{\partial \pi_{l}}+\frac{2\left(r_{a}-r_{i}\right)}{\rho_{i}^{r}} \frac{\partial^{2} r_{a}}{\partial \pi_{k} \partial \pi_{l}} \\
\frac{\partial^{2} M_{i a}^{\phi}}{\partial \pi_{k} \partial \pi_{l}} & =\frac{\cos \left(\phi_{a}-\phi_{i}\right)}{\rho_{i}^{\phi}} \frac{\partial \phi_{a}}{\partial \pi_{k}} \frac{\partial \phi_{a}}{\partial \pi_{l}}+\frac{\left.\sin \left(\phi_{a}-\phi_{i}\right)\right)}{\rho_{i}^{\phi}} \frac{\partial^{2} \phi_{a}}{\partial \pi_{k} \partial \pi_{l}} \\
\frac{\partial^{2} M_{i a}^{z}}{\partial \pi_{k} \partial \pi_{l}} & =\frac{2}{\rho_{i}^{z}} \frac{\partial z_{a}}{\partial \pi_{k}} \frac{\partial z_{a}}{\partial \pi_{l}}+\frac{2\left(z_{a}-z_{i}\right)}{\rho_{i}^{z}} \frac{\partial^{2} z_{a}}{\partial \pi_{k} \partial \pi_{l}}
\end{aligned}
$$

These equations are common to all expressions, exact or approximations, used to describe a track. The next two sections contain the expressions needed to complete these equations and it starts with the straight line approximation.

\section{B.1 Straight line approximation}

As described in section 3.1 a high energetic track that starts in vertex can be approximated with a straight line in the $r-\phi$ plane. To obtain a structure which is easy to compute, equations 6-9 are rewritten as three equations. The equations 
obtained from this are, as can be seen below, simple and the complete lack of trigonometric expressions makes them fast to calculate.

$$
\begin{aligned}
& \phi_{a}=\frac{2 r_{i} \kappa_{a}+\kappa_{a}^{2} \phi_{i}+4 \phi_{0 a}^{\prime}}{4+\kappa_{a}^{2}} \\
& r_{a}=\frac{2}{\kappa_{a}}\left(\phi_{a}-\phi_{0 a}^{\prime}\right) \\
& z_{a}=z_{0 a}+2 \gamma_{a} \operatorname{sgn}\left(\kappa_{a}\right)\left(\phi_{a}-\phi_{0 a}^{\prime}\right) \\
& \frac{\partial \phi_{a}}{\partial \kappa_{a}}=8 \frac{r_{i}+\kappa_{a} \phi_{i}-\kappa_{a} \phi_{0 a}^{\prime}-\frac{1}{4} \kappa_{a}^{2} r_{i}}{\left(4+\kappa_{a}^{2}\right)^{2}} \\
& \frac{\partial \phi_{a}}{\partial \phi_{0 a}^{\prime}}=\frac{4}{4+\kappa_{a}^{2}} \\
& \frac{\partial r_{a}}{\partial \kappa_{a}}=-\frac{2}{\kappa_{a}^{2}}\left(\phi_{a}-\phi_{0 a}^{\prime}\right)+\frac{2}{\kappa_{a}} \frac{\partial \phi_{a}}{\partial \kappa_{a}} \\
& \frac{\partial r_{a}}{\partial \phi_{0 a}^{\prime}}=\frac{2}{\kappa_{a}}\left(\frac{\partial \phi_{a}}{\partial \phi_{0 a}^{\prime}}-1\right) \\
& \frac{\partial z_{a}}{\partial \kappa_{a}}=2 \gamma_{a} \operatorname{sgn}\left(\kappa_{a}\right) \frac{\partial \phi_{a}}{\partial \kappa_{a}} \\
& \frac{\partial z_{a}}{\partial \phi_{0 a}^{\prime}}=2 \gamma_{a} \operatorname{sgn}\left(\kappa_{a}\right)\left(\frac{\partial \phi_{a}}{\partial \phi_{0 a}^{\prime}}-1\right) \\
& \frac{\partial z_{a}}{\partial \gamma_{a}}=2 \operatorname{sgn}\left(\kappa_{a}\right)\left(\phi_{a}-\phi_{0 a}^{\prime}\right) \\
& \frac{\partial z_{a}}{\partial z_{0 a}}=1 \\
& \frac{\partial^{2} \phi_{a}}{\partial \kappa_{a}^{2}}=4 \frac{6 \kappa_{a}^{2}\left(\phi_{0 a}^{\prime}-\phi_{i}\right)+4\left(2 \phi_{i}-\phi_{0 a}^{\prime}-3 \kappa_{a} r_{i}\right)+\kappa_{a}^{3} r_{i}}{\left(4+\kappa_{a}^{2}\right)^{3}} \\
& \frac{\partial^{2} \phi_{a}}{\partial \kappa_{a} \partial \phi_{0 a}^{\prime}}=-\frac{8 \kappa_{a}}{\left(4+\kappa_{a}^{2}\right)^{2}} \\
& \frac{\partial^{2} \phi_{a}}{\partial \phi_{0 a}^{\prime 2}}=0 \\
& \frac{\partial^{2} r_{a}}{\partial \kappa_{a}^{2}}=\frac{2}{\kappa_{a}}\left(\frac{2}{\kappa_{a}^{2}}\left(\phi_{a}-\phi_{0 a}^{\prime}\right)-\frac{2}{\kappa_{a}} \frac{\partial \phi_{a}}{\partial \kappa_{a}}+\frac{\partial^{2} \phi_{a}}{\partial \kappa_{a}^{2}}\right) \\
& \frac{\partial^{2} r_{a}}{\partial \kappa_{a} \partial \phi_{0 a}^{\prime}}=-\frac{2}{\kappa_{a}^{2}}\left(\frac{\partial \phi_{a}}{\partial \phi_{0 a}^{\prime}}-1\right)+\frac{2}{\kappa_{a}} \frac{\partial^{2} \phi_{a}}{\partial \kappa_{a} \partial \phi_{0 a}^{\prime}} \\
& \frac{\partial^{2} r_{a}}{\partial \phi_{0 a}^{\prime 2}}=0
\end{aligned}
$$




$$
\begin{aligned}
& \frac{\partial^{2} z_{a}}{\partial \kappa_{a}^{2}}=2 \gamma_{a} \operatorname{sgn}\left(\kappa_{a}\right) \frac{\partial^{2} \phi_{a}}{\partial \kappa_{a}^{2}} \\
& \frac{\partial^{2} z_{a}}{\partial \kappa_{a} \partial \phi_{0 a}^{\prime}}=2 \gamma_{a} \operatorname{sgn}\left(\kappa_{a}\right) \frac{\partial^{2} \phi_{a}}{\partial \kappa_{a} \partial \phi_{0 a}^{\prime}} \\
& \frac{\partial^{2} z_{a}}{\partial \kappa_{a} \partial \gamma_{a}}=2 \operatorname{sgn}\left(\kappa_{a}\right) \frac{\partial \phi_{a}}{\partial \kappa_{a}} \\
& \frac{\partial^{2} z_{a}}{\partial \kappa_{a} \partial \gamma_{a}}=0 \\
& \frac{\partial^{2} z_{a}}{\partial \phi_{0 a}^{\prime 2}}=2 \gamma_{a} \operatorname{sgn}\left(\kappa_{a}\right) \frac{\partial^{2} \phi_{a}}{\partial \phi_{0 a}^{\prime 2}} \\
& \frac{\partial^{2} z_{a}}{\partial \phi_{0 a}^{\prime} \partial \gamma_{a}}=2 \operatorname{sgn}\left(\kappa_{a}\right)\left(\frac{\partial \phi_{a}}{\partial \phi_{0 a}^{\prime}}-1\right) \\
& \frac{\partial^{2} z_{a}}{\partial \phi_{0 a}^{\prime} \partial z_{0 a}}=\frac{\partial^{2} z_{a}}{\partial \gamma_{a}^{2}}=\frac{\partial^{2} z_{a}}{\partial \gamma_{a} \partial z_{0 a}}=\frac{\partial^{2} z_{a}}{\partial z_{0 a}^{2}}=0
\end{aligned}
$$

\section{B.2 Exact expression}

The equations corresponding to the exact expressions in equations 2-5 are of course much longer and more computationally demanding, but if the expressions that describe the circle are rewritten as

$$
\begin{aligned}
& h_{1}=1+\kappa_{a} r_{0 a}-\kappa_{a} r_{i} \cos \left(\phi_{0 a}-\phi_{i}\right) \\
& h_{2}=\kappa_{a} r_{i} \sin \left(\phi_{0 a}-\phi_{i}\right) \\
& \cos (t)=\frac{h_{1}}{\sqrt{\left|h_{1}\right|^{2}+\left|h_{2}\right|^{2}}} \\
& f=r_{0 a}+\frac{1}{\kappa_{a}}(1-\cos (t)) \\
& g=\frac{1}{\kappa_{a}} \sin (t) \\
& r_{a}=\sqrt{|f|^{2}+|g|^{2}} \\
& \phi_{a}=-\arccos \frac{f}{r_{a}}+\phi_{0 a}
\end{aligned}
$$

one sees that they have several factors in common. There will actually only be 5 trigonometric expressions used $\cos (t), \sin (t), \cos \left(\phi_{0 a}-\phi_{a}\right), \sin \left(\phi_{0 a}-\phi_{a}\right)$ and the expression in $\phi_{a}$. These equations are used to obtain the first derivatives, 
where $\pi=\left\{\kappa_{a}, r_{0 a}, \phi_{a}\right\}$

$$
\begin{aligned}
& \frac{\partial h_{1}}{\partial \pi_{l}}=r_{0 a} \frac{\partial \kappa_{a}}{\partial \pi_{l}}+\kappa_{a} \frac{\partial r_{0 a}}{\partial \pi_{l}}-\frac{\partial \kappa_{a}}{\partial \pi_{l}} r_{i} \cos \left(\phi_{0 a}-\phi_{i}\right)+\kappa_{a} r_{i} \sin \left(\phi_{0 a}-\phi_{i}\right) \frac{\partial \phi_{0 a}}{\partial \pi_{l}} \\
& \frac{\partial h_{2}}{\partial \pi_{l}}=\frac{\partial \kappa_{a}}{\partial \pi_{l}} r_{i} \sin \left(\phi_{0 a}-\phi_{i}\right)+\kappa_{a} r_{i} \cos \left(\phi_{0 a}-\phi_{i}\right) \frac{\partial \phi_{0 a}}{\partial \pi_{l}} \\
& \frac{\partial \cos (t)}{\partial \pi_{l}}=\frac{h_{2}^{2} \frac{\partial h_{1}}{\partial \pi_{l}}-h_{1} h_{2} \frac{\partial h_{2}}{\partial \pi_{l}}}{\left(h_{1}^{2}+h_{2}^{2}\right)^{3 / 2}} \\
& \frac{\partial f}{\partial \pi_{l}}=\frac{\partial r_{0 a}}{\partial \pi_{l}}+\frac{\partial \kappa_{a}^{-1}}{\partial \pi_{l}}(1-\cos (t))-\frac{1}{\kappa_{a}} \frac{\partial \cos (t)}{\partial \pi_{l}} \\
& \frac{\partial g}{\partial \pi_{l}}=\frac{\partial \kappa_{a}^{-1}}{\partial \pi_{l}} \sin (t)-\frac{1}{\kappa_{a}} \frac{\cos (t)}{\sin (t)} \frac{\partial \cos (t)}{\partial \pi_{l}} \\
& \frac{\partial r_{a}}{\partial \pi_{l}}=\frac{f \frac{\partial f}{\partial \pi_{l}}+g \frac{\partial g}{\partial \pi_{l}}}{r_{a}} \\
& \frac{\partial \phi_{a}}{\partial \pi_{l}}=\frac{r_{a} \frac{\partial f}{\partial \pi_{l}}-f \frac{\partial r_{a}}{\partial \pi_{l}}}{|g| r_{a}}+\frac{\partial \phi_{0 a}}{\partial \pi_{l}}
\end{aligned}
$$

which gives the second derivatives

$$
\begin{aligned}
\frac{\partial^{2} h_{1}}{\partial \pi_{k} \partial \pi_{l}}= & \left(\frac{\partial \kappa_{a}}{\partial \pi_{l}} \frac{\partial r_{0 a}}{\partial \pi_{k}}+\frac{\partial \kappa_{a}}{\partial \pi_{k}} \frac{\partial r_{0 a}}{\partial \pi_{l}}\right)+\left(\frac{\partial \kappa_{a}}{\partial \pi_{l}} \frac{\partial \phi_{0 a}}{\partial \pi_{k}}+\frac{\partial \kappa_{a}}{\partial \pi_{k}} \frac{\partial \phi_{0 a}}{\partial \pi_{l}}\right) r_{i} \sin \left(\phi_{0 a}-\phi_{i}\right)+ \\
& +\kappa_{a} r_{i} \cos \left(\phi_{0 a}-\phi_{i}\right) \frac{\partial \phi_{0 a}}{\partial \pi_{k}} \frac{\partial \phi_{0 a}}{\partial \pi_{l}} \\
\frac{\partial^{2} h_{2}}{\partial \pi_{k} \partial \pi_{l}}= & \left(\frac{\partial \kappa_{a}}{\partial \pi_{l}} \frac{\partial \phi_{0 a}}{\partial \pi_{k}}+\frac{\partial \kappa_{a}}{\partial \pi_{k}} \frac{\partial \phi_{0 a}}{\partial \pi_{l}}\right) r_{i} \cos \left(\phi_{0 a}-\phi_{i}\right)-\kappa_{a} r_{i} \sin \left(\phi_{0 a}-\phi_{i}\right) \frac{\partial \phi_{0 a}}{\partial \pi_{k}} \frac{\partial \phi_{0 a}}{\partial \pi_{l}} \\
\frac{\partial^{2} \cos t}{\partial \pi_{k} \partial \pi_{l}}= & \frac{\left(2 h_{2} \frac{\partial h_{2}}{\partial \pi_{k}} \frac{\partial h_{1}}{\partial \pi_{l}}+\left|h_{2}\right|^{2} \frac{\partial^{2} h_{1}}{\partial \pi_{k} \partial \pi_{l}}-h_{2} \frac{\partial h_{1}}{\partial \pi_{k}} \frac{\partial h_{2}}{\partial \pi_{l}}-h_{1} \frac{\partial h_{2}}{\partial \pi_{k}} \frac{\partial h_{2}}{\partial \pi_{l}}-\right.}{\left(\sqrt{\left|h_{1}\right|^{2}+\left|h_{2}\right|^{2}}\right)^{5}} \\
\left.-h_{1} h_{2} \frac{\partial^{2} h_{2}}{\partial \pi_{k} \partial \pi_{l}}\right)\left(\sqrt{\left|h_{1}\right|^{2}+\left|h_{2}\right|^{2}}\right)^{2}-3\left(h_{2}^{2} \frac{\partial h_{1}}{\partial \pi_{l}}-h_{1} h_{2} \frac{\partial h_{2}}{\partial \pi_{l}}\right)\left(h_{1} \frac{\partial h_{1}}{\partial \pi_{k}}+h_{2} \frac{\partial h_{2}}{\partial \pi_{k}}\right) &
\end{aligned}
$$




$$
\begin{aligned}
& \frac{\partial^{2} f}{\partial \pi_{k} \partial \pi_{l}}= \frac{\partial^{2} \kappa_{a}^{-1}}{\partial \pi_{k} \partial \pi_{l}}(1-\cos t)-\frac{\partial \kappa_{a}^{-1}}{\partial \pi_{l}} \frac{\partial \cos t}{\partial \pi_{k}}-\frac{\partial \kappa_{a}^{-1}}{\partial \pi_{k}} \frac{\partial \cos t}{\partial \pi_{l}}-\frac{1}{\kappa_{a}} \frac{\partial^{2} \cos t}{\partial \pi_{k} \partial \pi_{l}} \\
& \frac{\partial^{2} g}{\partial \pi_{k} \partial \pi_{l}}= \frac{\partial^{2} \kappa_{a}^{-1}}{\partial \pi_{k} \partial \pi_{l}} \sin t-\frac{\partial \kappa_{a}^{-1}}{\partial \pi_{l}} \frac{\cos t}{\sin t} \frac{\partial \cos t}{\partial \pi_{k}}-\frac{\partial \kappa_{a}^{-1}}{\partial \pi_{k}} \frac{\cos t}{\sin t} \frac{\partial \cos t}{\partial \pi_{l}}- \\
&-\frac{1}{\kappa_{a}}\left(\frac{\cos t}{\sin t} \frac{\partial^{2} \cos t}{\partial \pi_{k} \partial \pi_{l}}+\frac{1}{\sin ^{3} t} \frac{\partial \cos t}{\partial \pi_{l}} \frac{\partial \cos t}{\partial \pi_{k}}\right) \\
& \frac{\partial^{2} r_{a}}{\partial \pi_{k} \partial \pi_{l}}= \frac{\left(\frac{\partial f}{\partial \pi_{k}} \frac{\partial f}{\partial \pi_{l}}+f \frac{\partial^{2} f}{\partial \pi_{k} \partial \pi_{l}}+\frac{\partial g}{\partial \pi_{k}} \frac{\partial g}{\partial \pi_{l}}+g \frac{\partial^{2} g}{\partial \pi_{k} \partial \pi_{l}}\right) r_{a}-\left(f \frac{\partial f}{\partial \pi_{l}}+g \frac{\partial g}{\partial \pi_{l}}\right) \frac{\partial r_{a}}{\partial \pi_{k}}}{r_{a}^{2}} \\
& \frac{\partial^{2} \phi_{a}}{\partial \pi_{k} \partial \pi_{l}}= \frac{\left(\frac{\partial^{2} f}{\partial \pi_{k} \partial \pi_{l}} r_{a}+\frac{\partial f}{\partial \pi_{l}} \frac{\partial r_{a}}{\partial \pi_{k}}-\frac{\partial f}{\partial \pi_{k}} \frac{\partial r_{a}}{\partial \pi_{l}}-f \frac{\partial^{2} r_{a}}{\partial \pi_{k} \partial \pi_{l}}\right)|g| r_{a}-}{\left(|g| r_{a}\right)^{2}} \\
&-\left(\frac{\partial f}{\partial \pi_{l}} r_{a}-f \frac{\partial r_{a}}{\partial \pi_{l}}\right)\left(\operatorname{sgng} \frac{\partial g}{\partial \pi_{k}} r_{a}+|g| \frac{\partial r_{a}}{\partial \pi_{k}}\right)
\end{aligned}
$$

The equations for the longitudinal movement are dependent on all five parameters, so with $\pi=\left\{\kappa_{a}, r_{0 a}, \phi_{a}, \gamma_{a}, z_{0 a}\right\}$ they become.

$$
\begin{aligned}
& z_{a}=z_{0 a}+\gamma_{a} t \\
& \frac{\partial z_{a}}{\partial \pi_{l}}=\frac{\partial z_{0 a}}{\partial \pi_{l}}+\frac{\partial \gamma_{a}}{\partial \pi_{l}} t-\gamma_{a} \frac{1}{\sin (t)} \frac{\partial \cos (t)}{\partial \pi_{l}} \\
& \frac{\partial^{2} z_{a}}{\partial \pi_{k} \partial \pi_{l}}=-\frac{1}{\sin (t)}\left(\frac{\partial \gamma_{a}}{\partial \pi_{k}} \frac{\partial \cos (t)}{\partial \pi_{l}}+\frac{\partial \gamma_{a}}{\partial \pi_{l}} \frac{\partial \cos (t)}{\partial \pi_{k}}\right)-\gamma_{a} \frac{\cos (t)}{\sin ^{3}(t)} \frac{\partial \cos (t)}{\partial \pi_{k}} \frac{\partial \cos (t)}{\partial \pi_{l}}- \\
& \quad-\gamma_{a} \frac{1}{\sin (t)} \frac{\partial^{2} \cos (t)}{\partial \pi_{k} \partial \pi_{l}}
\end{aligned}
$$

From these equations approximations can be made to handle special situations, but if this is not possible they can be used anywhere in the detector in their original form.

\section{B.2.1 Vertex}

The tracks that originate from the collision in the beam pipe (about $90 \%$ of all !!) have a known initial position within the error $\sigma_{v}^{2} \equiv \rho_{v}$. This can be used to make an additional hit since all parameters in the above equations are known in 
this case.

$M_{v a}=\frac{\left|r_{0 a}\right|^{2}}{\rho_{v}^{r}}+\frac{\left(1-\cos \left(\frac{\pi}{2}\left(\operatorname{sgn}\left(r_{0 a}\right)-1\right)+\phi_{0 a}-\phi_{v}\right)\right)}{\rho_{v}^{\phi}}+\frac{\left(z_{0 a}-z_{v}\right)^{2}}{\rho_{v}^{z}}$

But the $\phi$ term can be neglected since $\rho_{v}^{\phi} \rightarrow \infty$ (see equation 12). So the expression is

$M_{v a}=\frac{\left|r_{0 a}\right|^{2}}{\rho_{v}^{r}}+\frac{\left(z_{0 a}-z_{v}\right)^{2}}{\rho_{v}^{z}}$

and the first derivatives become

$\frac{\partial M_{v a}}{\partial r_{0 a}}=\frac{2 r_{0 a}}{\rho_{v}^{r}}$
$\frac{\partial M_{v a}}{\partial z_{0 a}}=\frac{2\left(z_{a}-z_{v}\right)}{\rho_{v}^{z}}$

This is the contribution to $\Delta \pi$ is since $V_{v a}=1 \forall a$. No decision-making is necessary, all these tracks pass through this hit. To just add this as a common hit might not serve its purpose since decisions could be made to disconnect a track from the vertex. The replacements that are done are

$\frac{\partial E_{e f f}}{\partial r_{0 a}} \rightarrow \frac{\partial E_{e f f}}{\partial r_{0 a}}+\frac{\partial M_{v a}}{\partial r_{0 a}}$

$\frac{\partial E_{e f f}}{\partial z_{0 a}} \rightarrow \frac{\partial E_{e f f}}{\partial z_{0 a}}+\frac{\partial M_{v a}}{\partial z_{0 a}}$

The contributions to the Hessian matrix are

$$
\begin{aligned}
\frac{\partial^{2} M_{v a}}{\partial r_{0 a}^{2}} & =\frac{2}{\rho_{v}^{r}} \\
\frac{\partial^{2} M_{v a}}{\partial z_{0 a}^{2}} & =\frac{2}{\rho_{v}^{z}}
\end{aligned}
$$

where the replacements are

$$
\frac{\partial^{2} E_{e f f}}{\partial r_{0 a}^{2}} \rightarrow \frac{\partial^{2} E_{e f f}}{\partial r_{0 a}^{2}}+\frac{\partial^{2} M_{v a}}{\partial r_{0 a}^{2}}
$$


$\frac{\partial^{2} E_{e f f}}{\partial z_{0 a}^{2}} \rightarrow \frac{\partial^{2} E_{e f f}}{\partial z_{0 a}^{2}}+\frac{\partial^{2} M_{v a}}{\partial z_{0 a}^{2}}$

This completes the structure that is used.

\section{References}

[1] Physics with proton beams I, Proceedings of the General Meeting on LHC Physics \& Detectors, Evian-les-Bains

[2] M.Ohlsson,C.Peterson and A. Yuille. Track finding with deformable templates - the elastic arms approach, Computer Physics Communications 71 (1992) 77 .

[3] J.Illingworth and J.Kittler, A survey of the Hough transform, Computer vision, graphics, and image processing 44, 87-116 (1988).

[4] M.Ohlsson. Extensions and explorations of the elastic arms algorithm, Computer Physics Communications 77 (1993) 19.

[5] Press,Flannery, Teukolsky and Vetterling, Numerical Recipes, Cambridge University Press, ISBN 0-521-38330-7

[6] R.Blankenbecler. Deformable templates-revisited and extended - with an oop implementation, Computer Physics Communications 81 (1994) 318-334

[7] R.Blankenbecler. A unified treatment of track reconstruction and particle identification, Computer Physics Communications 81 (1994) 335-342

[8] I.R.Kenyon, Elementary Particle Physics, ISBN 0-7102-1234-8.

[9] M.Ohlsson. Clustering Noisy Data with Deterministic Annealing, LU TP 93-28, December 1993

[10] J.Hertz,A.Krogh,R.G.Palmer, Introduction to the theory of neural computation, ISBN 0-201-5160-1. 